\title{
Ginseng Is Useful to Enhance Cardiac Contractility in Animals
}

\author{
Jia-Wei Lin, ${ }^{1}$ Yih-Giun Cherng, ${ }^{2}$ Li-Jen Chen, ${ }^{3}$ Ho-Shan Niu, ${ }^{4}$ \\ Chen Kuei Chang, ${ }^{1}$ and Chiang-Shan $\mathrm{Niu}^{4}$ \\ ${ }^{1}$ Department of Neurosurgery, Taipei Medical University-Shuang Ho Hospital and College of Medicine, Taipei Medical University, \\ Taipei 10361, Taiwan \\ ${ }^{2}$ Department of Anesthesiology, Taipei Medical University-Shuang Ho Hospital and College of Medicine, Taipei Medical University, \\ Taipei 10361, Taiwan \\ ${ }^{3}$ Institute of Basic Medical Sciences, College of Medicine, National Cheng Kung University, Tainan 70101, Taiwan \\ ${ }^{4}$ Department of Nursing, Tzu Chi College of Technology, Hualien City 97005, Taiwan
}

Correspondence should be addressed to Chiang-Shan Niu; ncs838@yahoo.com.tw

Received 7 December 2013; Accepted 25 December 2013; Published 4 February 2014

Academic Editor: Juei-Tang Cheng

Copyright (C) 2014 Jia-Wei Lin et al. This is an open access article distributed under the Creative Commons Attribution License, which permits unrestricted use, distribution, and reproduction in any medium, provided the original work is properly cited.

\begin{abstract}
Ginseng has been shown to be effective on cardiac dysfunction. Recent evidence has highlighted the mediation of peroxisome proliferator-activated receptors (PPARs) in cardiac function. Thus, we are interested to investigate the role of PPAR $\delta$ in ginsenginduced modification of cardiac contractility. The isolated hearts in Langendorff apparatus and hemodynamic analysis in catheterized rats were applied to measure the actions of ginseng ex vivo and in vivo. In normal rats, ginseng enhanced cardiac contractility and hemodynamic $d P / d t_{\max }$ significantly. Both actions were diminished by GSK0660 at a dose enough to block PPAR . However, ginseng failed to modify heart rate at the same dose, although it did produce a mild increase in blood pressure. Data of intracellular calcium level and Western blotting analysis showed that both the PPAR $\delta$ expression and troponin I phosphorylation were raised by ginseng in neonatal rat cardiomyocyte. Thus, we suggest that ginseng could enhance cardiac contractility through increased PPAR $\delta$ expression in cardiac cells.
\end{abstract}

\section{Introduction}

Ginseng varieties have been garnering increasing interest recently for their effects on the cardiovascular system [1]. It has been demonstrated that ginseng could prevent cardiac hypertrophy and heart failure through a mechanism likely involving the prevention of calcineurin activation [2] and the latter representing a key factor for myocardial hypertrophy and remodeling $[3,4]$.

Peroxisome proliferator-activated receptors (PPARs) are ligand-activated transcriptional factors that regulate the expression of genes involved in lipid metabolism and inflammation [5]. Three subtypes of PPARs, PPAR $\alpha, \operatorname{PPAR} \gamma$, and PPAR $\delta$, have been shown to modulate the expressions of various genes to exert bioactivity [5]. PPAR $\alpha$ is relatively abundant in tissues with a high oxidative capacity, such as the liver and heart, whereas PPAR $\gamma$ is confined to a limited number of tissues, primarily adipose tissue $[5,6]$. The ubiquitously expressed PPAR $\delta$ enhances lipid catabolism in adipose tissue and muscles [5], and $\operatorname{PPAR} \delta$-dependent maintenance of inotropic function is crucial for cardiomyocytes [7-9]. Deletion of cardiac PPAR $\delta$ is accompanied by decreased contraction, increased left ventricular end-diastolic pressure, and lowered cardiac output and leads to decreased contraction and increased incidence of cardiac failure [7].

It has been identified that cardiac agent, such as digoxin and dobutamine, can restore the cardiac contractility in diabetic rats [10-12]. Also, cardiac agent improved cardiac contraction in STZ-diabetic rats which is associated with a marked increase in cardiac PPAR $\delta$ expression [13].

Thus, we are interested to screen the effect of ginseng on cardiac contractility and investigate the mediation of $\operatorname{PPAR} \delta$ in this action of ginseng. Using the isolated hearts and animals in addition to cultured cardiac cells, the main aim of 
the present study is to clarify if ginseng can enhance cardiac contractility through increased $\operatorname{PPAR} \delta$ expression or not.

\section{Materials and Methods}

2.1. Materials. GSK0660 (a specific PPAR $\delta$ antagonist) was purchased from Santa Cruz Biotechnology, Inc. (Santa Cruz, CA, USA). The fluorescent probe, fura-2, was the product of Molecular Probes (Eugene, OR, USA). Antibodies specific to $\operatorname{PPAR} \delta$, cardiac troponin I (TnI) or phospho-troponin I (p-TnI) (Ser 23/24), were obtained from Cell Signaling Technology (Beverly, MA, USA).

2.2. Preparation of Fermented Panax ginseng (P. ginseng). Panax ginseng extract (GINST) used in the present study was provided by Bing-Han Pharmaceutics (Hsin-Yin, Tainan Shang, Taiwan). Briefly, dried Panax ginseng (1 kg) was extracted with $5 \mathrm{~L}$ of $50 \%$ aqueous ethanol at $85^{\circ} \mathrm{C}$ and concentrated in vacuous to obtain dark brown syrup. The dark brown syrup then was mixed with starch to generate the ginseng powder. The powder containing 95\% P. ginseng root and 5\% starch was dissolved in saline solution for oral administration at the desired doses.

2.3. Animals. Male Wistar rats, weighing from 200 to $250 \mathrm{~g}$, were obtained from the Animal Center of National Cheng Kung University Medical College. All experiments were performed under anesthesia with $2 \%$ isoflurane and all efforts were made to minimize suffering. The animal experiments were approved and conducted in accordance with local institutional guidelines for the care and use of laboratory animals in Chi-Mei Medical Center (no. 100052307) and performed according to the Guide for the Care and Use of Laboratory Animals as well as the guidelines of the Animal Welfare Act.

2.4. Drug Administration. Animals were randomly assigned into three groups: (I) the control group $(n=8)$ treated with the vehicle, saline ( $0.9 \%$ sodium chloride, orally); (II) the ginseng (Gin) group (orally. $n=8$ ) treated by oral administration with ginseng powder at $400 \mathrm{mg} / \mathrm{kg}$ for 7 days as described previously [14], and (III) the ginseng + GSK0660 $($ Gin + GSK) group $(n=8)$ treated with ginseng powder at effective dose $(400 \mathrm{mg} / \mathrm{kg}$, orally) according to previous report [14] and GSK0660 at effective dose (3 mg/kg, i.v.) [15] for 7 days. At the end of experiment, hearts of each group were dissected out for Western blotting analysis and Realtime reverse transcription-polymerase chain reaction.

2.5. Langendorff Apparatus for Isolated Heart Determination. The experiment was performed according to a previous description [16]. The rats were sacrificed under anesthesia with 3\% isoflurane and their hearts were excised rapidly and rinsed by immersion in ice-cold Krebs-Henseleit buffer (KHB) (mM: $\mathrm{NaCl} 118.5, \mathrm{KCl} 4.7, \mathrm{MgSO}_{4} 1.2, \mathrm{CaCl}_{2} \quad 1.8$, $\mathrm{NaHCO}_{3}$ 25.0, and glucose 11.0 at $\mathrm{pH}$ 7.35). The hearts were mounted in the Langendorff apparatus and continuously perfused with warm $\left(37^{\circ} \mathrm{C}\right)$ and oxygenated $\left(95 \% \mathrm{O}_{2}\right.$, $\left.5 \% \mathrm{CO}_{2}\right) \mathrm{KHB}$ at a constant pressure of $70 \mathrm{mmHg}$. The organ chamber temperature was maintained at $37^{\circ} \mathrm{C}$ during the experiment. A water-filled latex balloon was inserted through an incision in the left atrium into the left ventricle via the mitral valve and adjusted to a left ventricular end-diastolic pressure (LVEDP) of 5-7 mmHg during initial equilibrium. The distal end of the catheter was connected to an iWorx 214 TM data acquisition system (Ladscrib 2.0 software, iWorx Systems, Inc., Dover, NH, USA) via a pressure transducer for continuous recording. Left ventricular systolic function was assessed by recording the left ventricular developed pressure (LVDP), which was defined as the difference between left ventricular end-systolic pressure (LVESP) and LVEDP.

\subsection{Catheterization for Hemodynamic $d P / d t$ Measurement.} This part of experiments was performed in rats under anesthesia with $2 \%$ isoflurane to the minimize suffering of animals. Temporary pacing leads were used for the shortterm study and were placed in the right atrium and RV apex. A venogram imaged in 2 different angulations (left anterior oblique $30^{\circ}$ and anteroposterior) was obtained to determine the anatomy of the coronary sinus venous system. An LV pacing electrode (IX-214; iWorx Systems, Inc., Dover, NH, USA) was placed either in the free wall region via the lateral or posterior vein or in the anterior region via the great cardiac vein. After femoral artery and venous puncture using the Seldinger technique [17], pressure transducer catheters were inserted into the heart to provide the RV, aortic, mean blood, and LV pressures. Pressure catheters and pacing leads were connected to an external pacing computer (iWorx Systems, Inc., Dover, NH, USA) to monitor the heart rate and to acquire hemodynamic signals. Body temperature of the rats was also maintained at $37.5^{\circ} \mathrm{C}$ throughout whole procedure.

2.7. Cell Cultures. Primary cultures of neonatal rat cardiomyocytes were prepared by the modification of a previously described method [18]. Briefly, under anesthesia with $3 \%$ isoflurane, the hearts of 1- to 2-day-old Wistar rats were excised, cut into 1-2 $\mathrm{mm}$ pieces, and predigested with trypsin to remove red blood cells. The heart tissue was then digested with $0.25 \%$ trypsin and $0.05 \%$ collagenase. The dissociated cells were placed in uncoated $100 \mathrm{~mm}$ dishes and incubated at $37^{\circ} \mathrm{C}$ in a $5 \% \mathrm{CO}_{2}$ incubator for at least $1 \mathrm{~h}$ to remove the nonmyocytic cells. This procedure caused fibroblasts to predominantly attach to the dishes while most of the cardiomyocytes remained in suspension. The cardiomyocyteenriched population was then collected and counted. The cells were cultured in Dulbecco/Vogt modified Eagle's minimal essential medium (DMEM) with $1 \mathrm{mmol} / \mathrm{L}$ pyruvate, $10 \%$ fetal bovine serum, 100 units $/ \mathrm{mL}$ penicillin, and 100 units/mL streptomycin. Over $95 \%$ of the collected cells were characterized as cardiomyocytes on the basis of the sarcomeric myosin content. On the second day, the medium was replaced. After 3 to 4 days in culture, the cells were used in the experiments. Stock solutions of ginseng and GSK0660 were prepared with DMSO (0.1\%). The cells were treated with $100 \mu \mathrm{g} / \mathrm{mL}$ ginseng for $24 \mathrm{~h}$ [14], washed twice with PBS, and removed by trypsinization. The cells were then collected and 
subjected to a protein expression assay. Additional treatments with GSK0660 $\left(10^{-6} \mathrm{M}\right)$ [19] were performed for 30 minutes before the ginseng treatment.

\subsection{Measurement of Intracellular Calcium Concentration.} The changes in intracellular calcium were detected using the fluorescent probe fura-2-AM [20]. The neonatal rat cardiomyocytes were placed in buffered physiological saline solution (PSS) containing $140 \mathrm{mM} \mathrm{NaCl}, 5.9 \mathrm{mM} \mathrm{KCl}, 1.2 \mathrm{mM}$ $\mathrm{CaCl}_{2}, 1.4 \mathrm{mM} \mathrm{MgCl} 2,11.5 \mathrm{mM}$ glucose, $1.8 \mathrm{mM} \mathrm{Na}_{2} \mathrm{HPO}_{4}$, and $10 \mathrm{mM}$ Hepes-Tris, to which was added $5 \mu \mathrm{M}$ fura-2$\mathrm{AM}$, and the solution was incubated for $1 \mathrm{~h}$ in humidified $5 \% \mathrm{CO}_{2}$ and $95 \%$ air at $37^{\circ} \mathrm{C}$. The cells were washed and incubated for an additional 30 minutes in PSS. The neonatal rat cardiomyocytes were inserted into a thermostatic $\left(37^{\circ} \mathrm{C}\right)$ cuvette containing $2 \mathrm{~mL}$ of calcium-free PSS. After recording the baseline value, ginseng $(100 \mu \mathrm{g} / \mathrm{mL})$ was added into the cuvette with/without GSK0660 $\left(10^{-6} \mathrm{M}\right)$ to detect the free intracellular calcium. The fluorescence was continuously recorded using a fluorescence spectrofluorometer (Hitachi F-2000, Tokyo, Japan). Values of $\left[\mathrm{Ca}^{2+}\right] \mathrm{i}$ were calculated from the ratio $R=\mathrm{F} 340 / \mathrm{F} 380$ by the formula $\left[\mathrm{Ca}^{2+}\right] \mathrm{i}=K d B$ $\left(R-R_{\min }\right) /\left(R_{\max }-R\right)$, where $K d$ was $225 \mathrm{nM}$, $\mathrm{F}$ was fluorescence, and $B$ was the ratio of the fluorescence of the free dye to that of the $\mathrm{Ca}^{2+}$-bound dye measured at $380 \mathrm{~nm}$. $R_{\max }$ and $R_{\min }$ were determined in separate experiments by using ginseng to equilibrate $\left[\mathrm{Ca}^{2+}\right] \mathrm{j}$ with ambient $\left[\mathrm{Ca}^{2+}\right]$ $\left(R_{\max }\right)$ and the addition of $0.1 \mathrm{mM} \mathrm{MnCl}_{2}$ and $1 \mathrm{mmol} / \mathrm{L}$ EGTA $\left(R_{\min }\right)$. Background autofluorescence was measured in unloaded cells and subtracted from all measurements.

2.9. Western Blotting Analysis. Protein was extracted from tissue homogenates and cell lysates using ice-cold radioimmunoprecipitation assay (RIPA) buffer supplemented with phosphatase and protease inhibitors $(50 \mathrm{mmol} / \mathrm{L}$ sodium vanadate, $0.5 \mathrm{mM}$ phenylmethylsulphonyl fluoride, $2 \mathrm{mg} / \mathrm{mL}$ aprotinin, and $0.5 \mathrm{mg} / \mathrm{mL}$ leupeptin). Protein concentrations were determined with a Bio-Rad protein assay (Bio-Rad Laboratories, Inc., Hercules, CA, USA). Total proteins (30 $\mu \mathrm{g}$ ) were separated by SDS/polyacrylamide gel electrophoresis (10\% acrylamide gel) using a Bio-Rad Mini-Protein II system. The protein was transferred to expanded polyvinylidene difluoride membranes (Pierce, Rockford, IL, USA) with a Bio-Rad Trans-Blot system. After transfer, the membranes were washed with PBS and blocked for $1 \mathrm{~h}$ at room temperature with $5 \%(\mathrm{w} / \mathrm{v})$ skimmed milk powder in PBS. The manufacturer's instructions were followed for the primary antibody reactions. Blots were incubated overnight at $4^{\circ} \mathrm{C}$ with an immunoglobulin-G polyclonal rabbit anti-mouse antibody (Affinity BioReagents, Inc., Golden, CO, USA) $(1: 500)$ in $5 \%(\mathrm{w} / \mathrm{v})$ skimmed milk powder dissolved in PBS/Tween 20 ( $0.5 \%$ by volume) to bind the target protein such as $\operatorname{PPAR} \delta$. The blots were incubated with goat polyclonal antibody $(1: 1000)$ to bind the actin, which served as the internal control. After the removal of the primary antibody, the blots were extensively washed with PBS/Tween 20 and then incubated for $2 \mathrm{~h}$ at room temperature with the appropriate peroxidase-conjugated secondary antibody diluted in 5\% (w/v) skimmed milk powder and dissolved in PBS/Tween 20. The blots were developed by autoradiography using an ECL-Western blotting system (Amersham International, Buckinghamshire, UK). The immunoblots of PPAR $\delta$ $(50 \mathrm{kDa})$, cardiac troponin I $(28 \mathrm{kDa})$, and phospho-troponin I $(28 \mathrm{kDa})$ were then quantified using a laser densitometer.

2.10. Statistical Analysis. Results were expressed as mean \pm SE of each group. Statistical analysis was carried out using ANOVA analysis and Newman-Keuls post hoc analysis. Statistical significance was considered at $P<0.05$.

\section{Results}

3.1. Effect of GW0742 on PPARS Expression and TnI Phosphorylation in the Heart of Rats. The rats treated with ginseng were used to identify changes in PPAR $\delta$ expression and TnI phosphorylation. The level of PPAR $\delta$ expression (Figure 1(a)) and TnI phosphorylation (Figure 1(b)) was markedly raised by ginseng at an effective concentration $(400 \mathrm{mg} / \mathrm{kg})$. In addition, this change was reversed by GSK0660 (3 mg/kg, i.v.) at a concentration that did not modify the level of PPAR $\delta$ expression and TnI phosphorylation (Figure 1).

\subsection{Effect of Ginseng on Cardiac Performance in the Isolated} Rat Heart. Ginseng at a sufficient dose to activate PPAR $\delta$ was used to treat the hearts isolated from rats. As shown in Figure 2, ginseng $(100 \mu \mathrm{g} / \mathrm{mL})$ increased cardiac contractility without changes in heart rate. Moreover, the cardiac tonic action of ginseng was diminished by GSK0660 $\left(10^{-6} \mathrm{M}\right)$ (Figure 2).

3.3. Effect of Ginseng on Cardiac Performance in the Anesthetized Rats. The $d P / d t_{\max }$ was significantly increased by ginseng after the treatment $(400 \mathrm{mg} / \mathrm{kg} /$ day, orally for 7 days) as described previously [14] in the anesthetized rats, compared with the vehicle-treated control. However, this effect disappeared in the rats receiving coadministration of GSK0660 at effective dose (3 mg/kg, i.v.) [15] (Figure 3(a)). Treatment of ginseng only did not modify the heart rate but produced a slight increase in blood pressure that was also blocked by GSK0660 (Figures 3(b) and 3(c)).

\subsection{Effect of Ginseng on Intracellular Calcium in Neonatal Rat Cardiomyocytes. The fluorescent probe, fura2-AM, was used to detect changes in intracellular calcium level in the neonatal rat cardiomyocytes, and ginseng at an effective concentra- tion $(100 \mu \mathrm{g} / \mathrm{mL})$ was found to increase the intracellular calcium level. This effect disappeared in the cardiomyocytes that were coincubated with ginseng and GSK0660 $\left(10^{-6} \mathrm{M}\right)$ (Figure 4(a)); however, incubation with GSK0660 alone did not affect the intracellular calcium level in the neonatal rat cardiomyocytes (Figure 4(a)).}

3.5. Effects of Ginseng on PPAR $\delta$ Expression and TnI Phosphorylation in Neonatal Rat Cardiomyocytes. The neonatal rat cardiomyocytes were applied to treat with ginseng for 


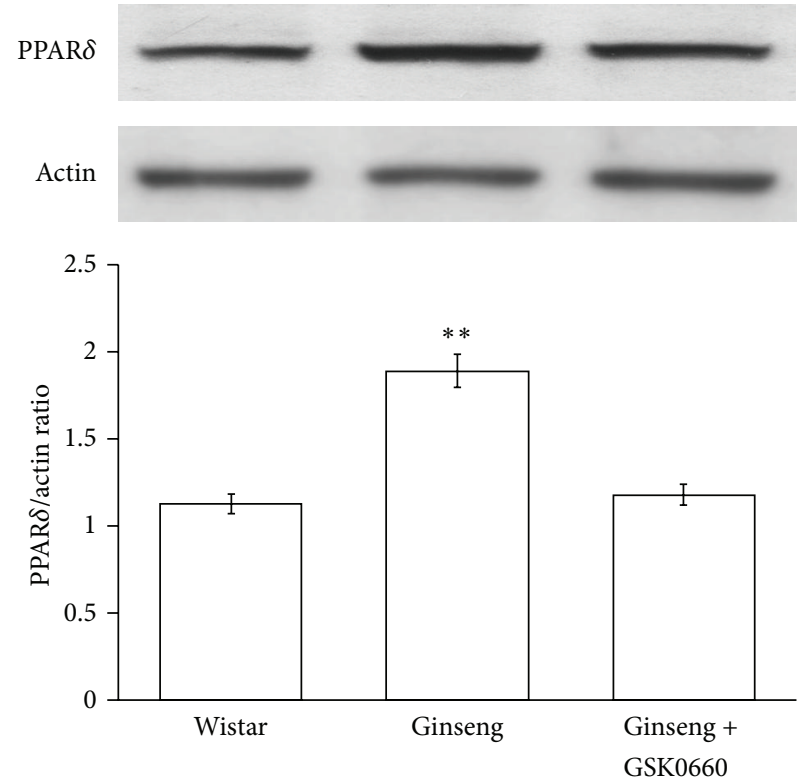

(a)

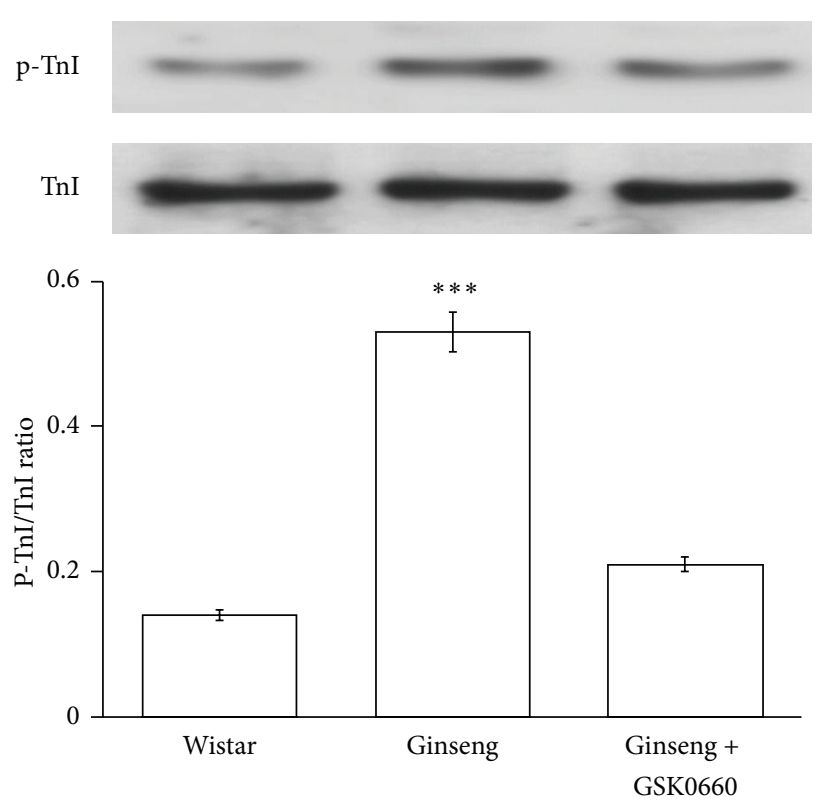

(b)

FIGURE 1: Effects of ginseng on PPAR $\delta$ expression and TnI phosphorylation in heart of rats. Changes of PPAR $\delta$ expression (a) and TnI phosphorylation (b) in the hearts of rats treated with ginseng. Rats were treated with ginseng ( $400 \mathrm{mg} / \mathrm{kg})$ for 7 days and hearts were then used to measure the protein level of PPAR $\delta$ expression and TnI phosphorylation using Western blotting analysis. All values are presented as mean $\pm \operatorname{SEM}(n=8) .{ }^{* *} P<0.01$ and ${ }^{* * *} P<0.001$ compared with normal rats.

identification of changes in PPAR $\delta$ expression and TnI phosphorylation. The levels of PPAR $\delta$ expression and TnI phosphorylation were markedly raised by ginseng at an effective concentration $(100 \mu \mathrm{g} / \mathrm{mL})$. Also, this effect was reversed by GSK0660 $\left(10^{-6} \mathrm{M}\right)$ at a concentration that did not modify the level of PPAR $\delta$ expression and TnI phosphorylation (Figure 4(b)).

\section{Discussion}

In the present study, we found that ginseng increased cardiac contractility but not heart rate in rats at the dose of $400 \mathrm{mg} / \mathrm{kg}$, orally. This dose is equal to human oral dose about $3871 \mathrm{mg} / \mathrm{kg}$ by using the US FDA HED (human equivalent dose) equation for calculation [21-23]. Increases in PPAR $\delta$ expression and TnI phosphorylation were also observed in the heart of ginseng-treated rats. In hearts isolated from rats, ginseng enhanced cardiac contractility and this action was diminished by GSK0660 at a concentration sufficient to block PPAR $\delta$. In the anesthetized rats, cardiac contraction $\left(d P / d t_{\max }\right)$ was also significantly increased by ginseng and this change was blocked by GSK0660. However, heart rate was not modified by ginseng at same dose. In the neonatal rat cardiomyocytes, ginseng increased cellular calcium levels, PPAR $\delta$ expression, and TnI phosphorylation. Thus, to the best of our knowledge, this is the first study to show that ginseng could increase cardiac contractility through activation of PPAR $\delta$.
In vivo and in vitro investigations have revealed a number of significant actions of ginsenosides and ginseng extracts in cardioprotection, such as reducing myocardial ischemiareperfusion induced damage via $\mathrm{NO}$ pathway in rats and mice [24], slowing down deterioration of cardiac contractions, preventing the development of arrhythmias [25], and relaxing the muscles of the aorta [26]. Also, it has been documented that ginseng increases cardiac lipid metabolism by enhancement of PPAR $\delta$ expression in the hearing [27]. In this study, we found that ginseng could increase PPAR $\delta$ expression and TnI phosphorylation. Also, this action of ginseng was abolished by specific PPAR $\delta$ antagonist. Mediation of PPAR $\delta$ in this action of ginseng can thus be considered.

It has been established that PPAR $\delta$ plays an important role in the regulation of cardiac performance [17-19]. In this study, we demonstrated that ginseng increases cardiac contractility without affecting heart rate. Also, this cardiac action of ginseng is reversed by blockade of PPAR $\delta$ using antagonist. Furthermore, activation of PPAR $\delta$ using ginseng enhanced cardiac contractility in the isolated hearts and the hemodynamic $d P / d t_{\max }$ in the rats. Both actions of ginseng were inhibited by GSK0660 at a concentration sufficient to block PPAR $\delta[27,28]$. The enhancement of cardiac contractility by ginseng through an activation of PPAR $\delta$ is then characterized.

A change in heart rate is the most serious side effect of cardiac agents $[29,30]$. In the present study, we showed that ginseng could enhance cardiac contractility without altering heart rate in isolated heart. In addition, ginseng generated 


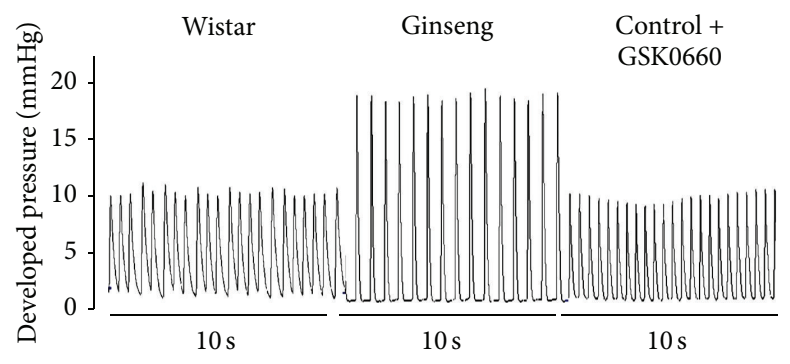

(a)

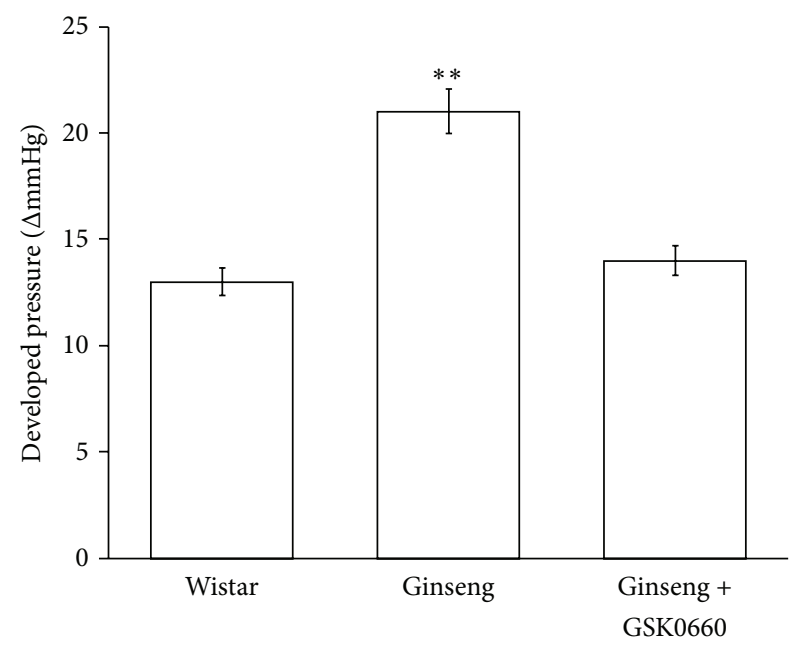

(b)

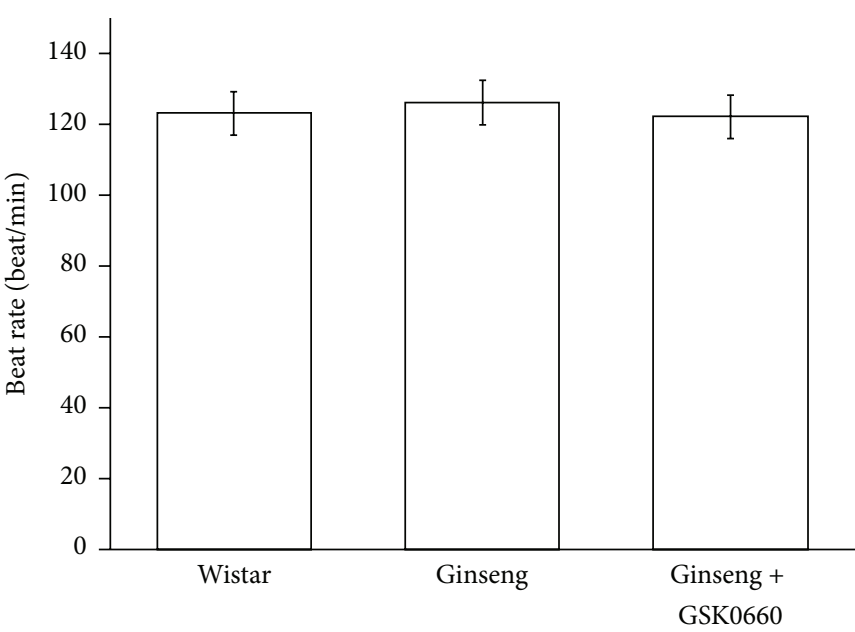

(c)

FIGURE 2: Effects of ginseng on cardiac performance in hearts isolated from rats. The representative picture shows the change in cardiac performance caused by ginseng in isolated hearts (a). Heart rate and cardiac contractility were recorded in isolated rat heart treated with ginseng or cotreated with ginseng + GSK0660. The changes in developed pressure (b) and beat rate (c) were recorded continuously throughout the whole experiment. All values are presented as mean $\pm \operatorname{SEM}(n=8) .{ }^{* *} P<0.01$ as compared to normal rats.

cardiac tonic action in animals without impacting the heart rate. Thus, ginseng can be used as cardiac agent at some doses without side effect of arrhythmia. Our previous studies have showed that activation of PPAR $\delta$ by cardiac agent may improve diabetic cardiomyopathy in type- 1 diabetic rats [28, 31]. Thus, ginseng seems helpful in the treatment and/or prevention of diabetic cardiomyopathy. However, a slight elevation of mean blood pressure was observed in the rats received ginseng. Thus, applying ginseng in patients with hypertension should be done carefully.

Troponin I (TnI) is known as an inhibitory unit of the troponin complex associated with thin filaments, and it inhibits actin-myosin interactions at the diastolic level of intracellular $\mathrm{Ca}^{2+}[32,33]$. Modulation of myofilament properties by alterations in TnI phosphorylation has been found to have a profound effect on cardiac contractility [34]. Phosphorylation of TnI has been shown to increase the crossbridge cycling rate, leading to an increase in power output $[33,34] . \mathrm{Ca}^{2+}$ is mainly involved in muscle contraction [3235]. Contraction of cardiac muscles relies upon interactions between ATP and $\mathrm{Ca}^{2+}$, both of which must be present in adequate amounts [36]. We observed that ginseng has the ability to increase the amount of intracellular calcium in cardiomyocytes, and this seemed to be related to the higher contractility of heart.

It has been shown that $\mathrm{TnI}$ phosphorylation most likely acts through an enhanced off rate during $\mathrm{Ca}^{2+}$ exchange with contractile protein, leading to an increase in cardiac output [36-40]. Consistent with this, we found that TnI phosphorylation was elevated in the neonatal rat cardiomyocytes exposed to ginseng. Thus, direct activation of PPAR $\delta$ by ginseng may result in a higher level of TnI phosphorylation. Both changes caused by ginseng in the cardiomyocytes may explain the increase in cardiac contractility.

The multiple cell signals are involved in cardiac contractility. It is not easy to speculate the potential mechanism(s) for this action of ginseng in the increase of cardiac contractility. It has been documented that ginsenoside, one of the active principles in ginseng, can improve systolic/diastolic function and enhance cardiac $d P / d t$ through the opening of mitochondrial adenosine triphosphate-sensitive potassium channels [41], reduction in oxidative stress via inhibition of glutathione [42], and augmentation of cellular calcium influx [43]. The increase of cellular calcium influx is consistent with 


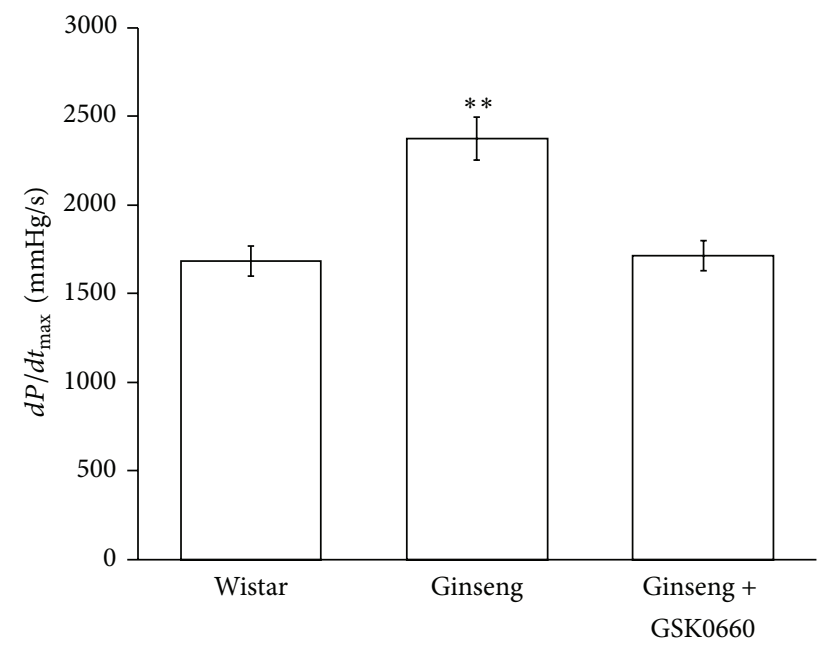

(a)

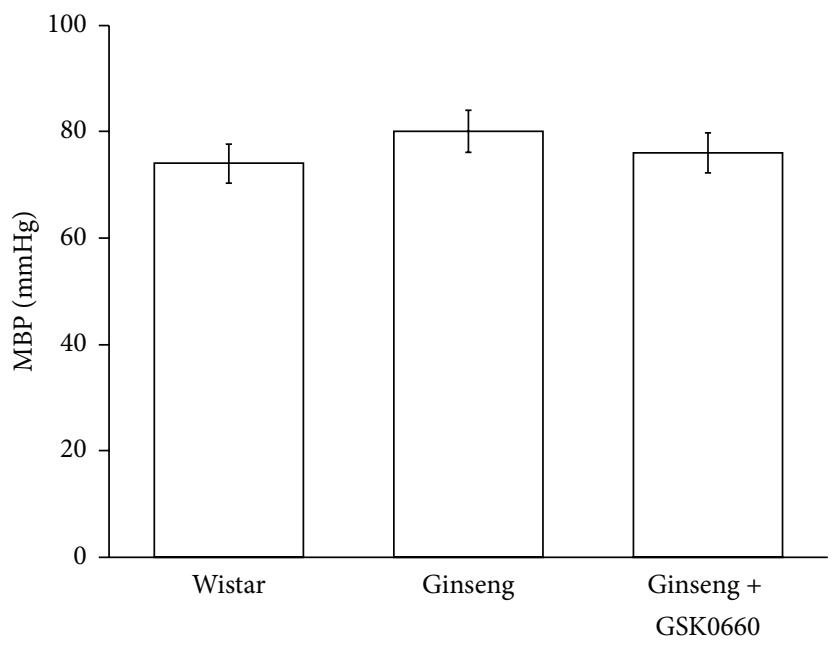

(b)

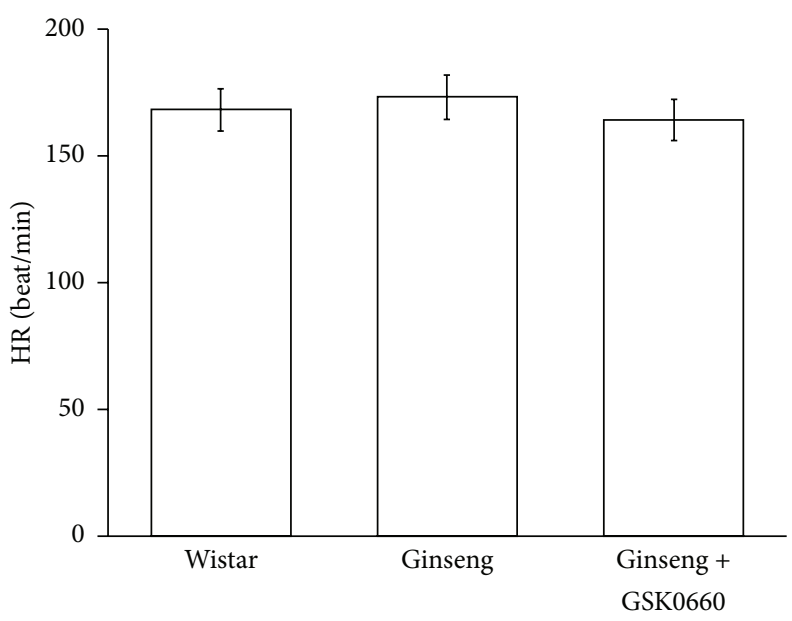

(c)

FIGURE 3: Effects of ginseng on cardiac performance in anesthetized rats. The effects of coadministration of ginseng and/or GSK0660 were investigated in the anesthetized rats. The changes in hemodynamic $d P / d t(\mathrm{a})$, mean blood pressure (MBP) (b), and heart rate (HR) (c) were recorded continuously throughout the whole experiment. All values are presented as mean $\pm \operatorname{SEM}(n=8) .{ }^{* *} P<0.01$ as compared to normal rats.

to our previous reports regarding $\operatorname{PPAR} \delta$ related cardiac tonic actions $[10,11,44]$.

Interestingly, ginseng enhanced cardiac contractility without altering heart rate in isolated heart. Also, ginseng generated cardiac tonic action in animals without impacting the heart rate. It has been demonstrated that PPAR $\delta$ agonist is not effective in cardiac conduction [45]. The possible explanation for this seems related to the absence of PPAR $\delta$ in cardiac conduction system. However, the real mechanism(s) for the lack of effect on heart rate caused by ginseng require(s) more investigations in the future.

The activation of PPAR $\delta$ by ginseng can enhance the cardiac contractility without altering heart rate. Thus, we suggest that ginseng is suitable for the treatment of heart failure. Using this agent, arrhythmia can be ignored in patients for treatment of heart failure.

\section{Conclusion}

According to these findings, we suggest that the activation of PPAR $\delta$ by ginseng increases intracellular calcium, which then results in cardiac troponin phosphorylation. Subsequently, the cardiac contractility is enhanced. Taken together, ginseng enhanced cardiac contractility through an increase in PPAR $\delta$ expression at the dose that did not modify the heart beating. Thus, ginseng could be developed as a good cardiac agent without the side effect on heart rate.

\section{Conflict of Interests}

The authors declare that there is no conflict of interests regarding the publication of this paper. 


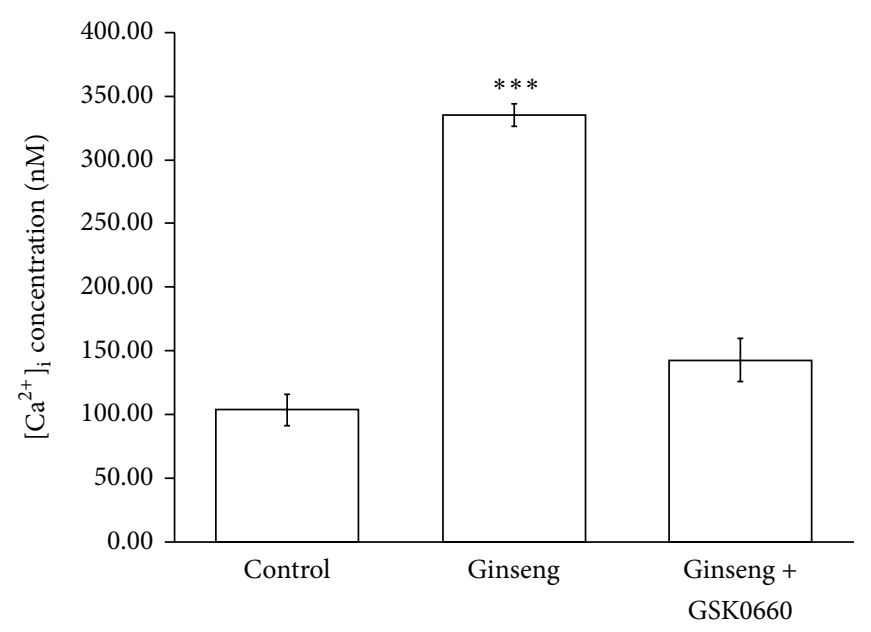

(a)

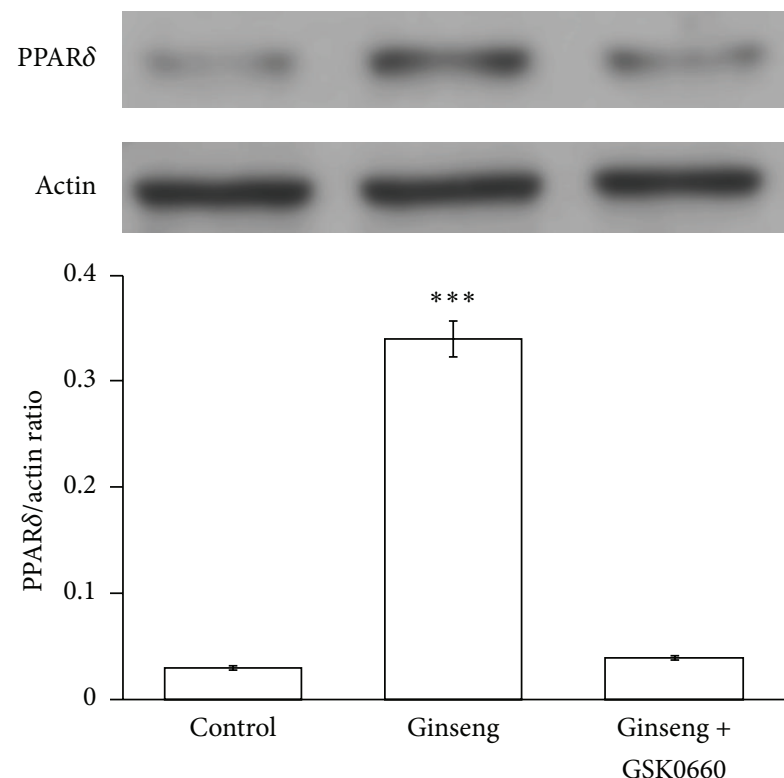

(b)

\section{p-TnI}

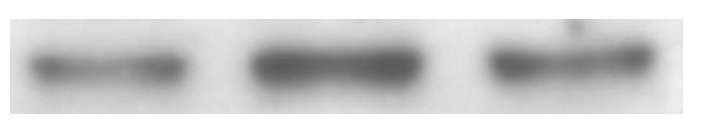

$\operatorname{TnI}$
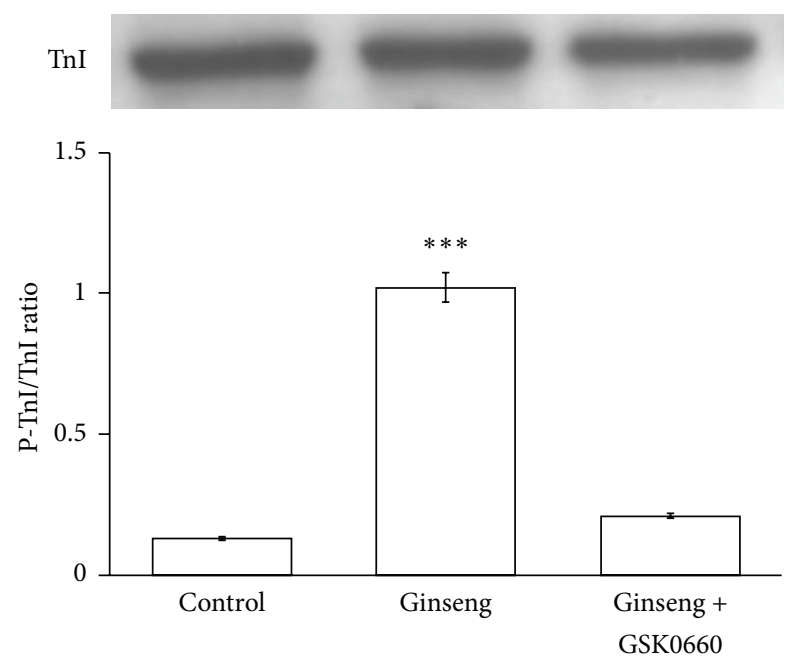

(c)

FIGURE 4: Effects of ginseng on intracellular calcium and TnI phosphorylation in neonatal rat cardiomyocytes. Changes in intracellular calcium were detected with fura-2 by using a fluorescence spectrofluorometer. The neonatal rat cardiomyocytes were placed in buffered physiological saline solution with $5 \mu \mathrm{M}$ of fura-2-AM and incubated for $1 \mathrm{~h}$. After recording the baseline value, ginseng was added into the cuvette with/without GSK0660 to detect the free intracellular calcium (a). Effects of ginseng on PPAR $\delta$ expression (b) and TnI phosphorylation (c) in the neonatal rat cardiomyocytes were indicated. Cells treated with ginseng for 24 hours were harvested to measure the protein level of PPAR $\delta$ expression and TnI phosphorylation using Western blotting analysis. All values are presented as mean \pm SEM $(n=8)$.

${ }^{* * *} P<0.001$ compared with the control group.

\section{Acknowledgments}

The authors thank Yang-Lian Yan and Yi-Zhi Chen for their assistance in their experiments. The present study was supported in part by a Grant (TCCT-1011A09) from Tzu Chi College of Technology, Hualien City, Taiwan.

\section{References}

[1] M. Karmazyn, M. Moey, and X. T. Gan, "Therapeutic potential of ginseng in the management of cardiovascular disorders," Drugs, vol. 71, no. 15, pp. 1989-2008, 2011.
[2] J. Guo, X. T. Gan, J. V. Haist et al., "Ginseng inhibits cardiomyocyte hypertrophy and heart failure via nhe-1 inhibition and attenuation of calcineurin activation," Circulation, vol. 4, no. 1, pp. 79-88, 2011.

[3] N. Frey and E. N. Olson, "Cardiac hypertrophy: the good, the bad, and the ugly," Annual Review of Physiology, vol. 65, pp. 4579, 2003.

[4] J. M. Berry, V. Le, D. Rotter et al., "Reversibility of adverse, calcineurin-dependent cardiac remodeling," Circulation Research, vol. 109, no. 4, pp. 407-417, 2011.

[5] Q. Yang and Y. Li, "Roles of PPARs on regulating myocardial energy and lipid homeostasis," Journal of Molecular Medicine, vol. 85, no. 7, pp. 697-706, 2007. 
[6] I. Issemann and S. Green, "Activation of a member of the steroid hormone receptor superfamily by peroxisome proliferators," Nature, vol. 347, no. 6294, pp. 645-650, 1990.

[7] L. Cheng, G. Ding, Q. Qin et al., "Cardiomyocyte-restricted peroxisome proliferator-activated receptor- $\delta$ deletion perturbs myocardial fatty acid oxidation and leads to cardiomyopathy," Nature Medicine, vol. 10, no. 11, pp. 1245-1250, 2004.

[8] L. Cheng, G. Ding, Q. Qin et al., "Peroxisome proliferatoractivated receptor $\delta$ activates fatty acid oxidation in cultured neonatal and adult cardiomyocytes," Biochemical and Biophysical Research Communications, vol. 313, no. 2, pp. 277-286, 2004.

[9] G. D. Barish, V. A. Narkar, and R. M. Evans, "PPAR $\delta$ : a dagger in the heart of the metabolic syndrome," Journal of Clinical Investigation, vol. 116, no. 3, pp. 590-597, 2006.

[10] M. T. Chou, S. H. Lo, K. C. Cheng, Y. X. Li, L. J. Chen, and J. T. Cheng, "Activation of $\beta$-adrenoceptors by dobutamine may induce a higher expression of peroxisome proliferatoractivated receptors $\delta$ (PPAR $\delta$ ) in neonatal rat cardiomyocytes," The Scientific World Journal, vol. 2012, Article ID 248320, 8 pages, 2012.

[11] Z.-C. Chen, B.-C. Yu, L.-J. Chen, K.-C. Cheng, H. J. Lin, and J.T. Cheng, "Characterization of the mechanisms of the increase in PPAR $\delta$ expression induced by digoxin in the heart using the H9c2 cell line," British Journal of Pharmacology, vol. 163, no. 2, pp. 390-398, 2011.

[12] Z. C. Chen, B. C. Yu, L. J. Chen, and J. T. Cheng, "Increase of peroxisome proliferator-activated receptor $\delta$ (PPAR $\delta$ ) by digoxin to improve lipid metabolism in the heart of diabetic rats," Hormone and Metabolic Research, vol. 45, no. 5, pp. 364371, 2013.

[13] B.-C. Yu, C.-K. Chang, H.-Y. Ou, K.-C. Cheng, and J.-T. Cheng, "Decrease of peroxisome proliferator-activated receptor delta expression in cardiomyopathy of streptozotocin-induced diabetic rats," Cardiovascular Research, vol. 80, no. 1, pp. 78-87, 2008.

[14] H.-X. Li, S.-Y. Han, X. Ma et al., “The saponin of red ginseng protects the cardiac myocytes against ischemic injury in vitro and in vivo," Phytomedicine, vol. 19, no. 6, pp. 477-483, 2012.

[15] I. Paterniti, E. Esposito, E. Mazzon et al., "Evidence for the role of peroxisome proliferator-activated receptor- $\beta / \delta$ in the development of spinal cord injury," Journal of Pharmacology and Experimental Therapeutics, vol. 333, no. 2, pp. 465-477, 2010.

[16] L. Sun, D.-L. Li, M. Zhao et al., "The role of muscarinic receptors in the beneficial effects of adenosine against myocardial reperfusion injury in rats," PLoS ONE, vol. 6, no. 11, Article ID e25618, 2011.

[17] S. I. Seldinger, "Catheter replacement of the needle in percutaneous arteriography: a new technique," Acta Radiologica, vol. 49, no. 434, pp. 47-52, 2008.

[18] Z. C. Chen, B. C. Yu, L. J. Chen, and J. T. Cheng, "Increase of peroxisome proliferator-activated receptor delta (PPARdelta) by digoxin to improve lipid metabolism in the heart of diabetic rats," Hormone and Metabolic Research, vol. 45, no. 5, pp. 364371, 2013.

[19] G. Cohen, Y. Riahi, O. Shamni et al., "Role of lipid peroxidation and PPAR- $\delta$ in amplifying glucose-stimulated insulin secretion," Diabetes, vol. 60, no. 11, pp. 2830-2842, 2011.

[20] H. A. Halaq and G. T. Haupert Jr., "Positive inotropic effects of the endogenous $\mathrm{Na}+/ \mathrm{K}+$-transporting ATPase inhibitor from the hypothalamus," Proceedings of the National Academy of Sciences of the United States of America, vol. 86, no. 24, pp. 10080-10084, 1989.
[21] I. Cavero, "10th annual meeting of the Safety Pharmacology Society: an overview," Expert Opinion on Drug Safety, vol. 10, no. 2, pp. 319-333, 2011.

[22] W. C. Buhles, "Compassionate use: a story of ethics and science in the development of a new drug," Perspectives in Biology and Medicine, vol. 54, no. 3, pp. 304-315, 2011.

[23] T. Keida, N. Hayashi, and M. Kawashima, "Application of the Food and Drug Administration (FDA) bioequivalent guidance of topical dermatological corticosteroid in yellow-skinned Japanese population: validation study using a chromameter," Journal of Dermatology, vol. 33, no. 10, pp. 684-691, 2006.

[24] H. Zhou, S. Z. Hou, P. Luo et al., "Ginseng protects rodent hearts from acute myocardial ischemia-reperfusion injury through GR/ER-activated RISK pathway in an endothelial NOS-dependent mechanism," Journal of Ethnopharmacology, vol. 135, no. 2, pp. 287-298, 2011.

[25] G. I. Scott, P. B. Colligan, B. H. Ren, and J. Ren, "Ginsenosides $\mathrm{Rb} 1$ and Re decrease cardiac contraction in adult rat ventricular myocytes: role of nitric oxide," British Journal of Pharmacology, vol. 134, no. 6, pp. 1159-1165, 2001.

[26] S. Y. Kang, V. B. Schini-Kerth, and N. D. Kim, "Ginsenosides of the protopanaxatriol group cause endothelium-dependent relaxation in the rat aorta," Life Sciences, vol. 56, no. 19, pp. 15771586, 1995.

[27] S. C. Kuo, P. M. Ku, L. J. Chen, H. S. Niu, and J. T. Cheng, "Activation of receptors $\delta$ (PPAR $\delta$ ) by agonist (GW0742) may enhance lipid metabolism in heart both in vivo and in vitro," Hormone and Metabolic Research, vol. 45, pp. 880-886, 2013.

[28] Y. Z. Cheng, L. J. Chen, W. J. Lee, M. F. Chen, H. Jung Lin, and J. T. Cheng, "Increase of myocardial performance by Rhodiolaethanol extract in diabetic rats," Journal of Ethnopharmacology, vol. 144, pp. 234-239, 2012.

[29] R. Marquardt, "Influence of dopamine and metaproterenol on serum free fatty acids in regard to induction of cardiac arrhythmia," Medizinische Welt, vol. 30, no. 18, pp. 707-708, 1979.

[30] E. T. Angelakos, M. P. King, and R. W. Millard, "Regional distribution of catecholamines in the hearts of various species," Annals of the New York Academy of Sciences, vol. 156, no. 1, pp. 219-240, 1969.

[31] S. C. Fan, B. C. Yu, Z. C. Chen, L. J. Chen, H. H. Chung, and J. T. Cheng, "The decreased expression of peroxisome proliferatoractivated receptors (PPAR) is reversed by digoxin in the heart of diabetic rats," Hormone and Metabolic Research, vol. 42, no. 9, pp. 637-642, 2010.

[32] I. Ohtsuki and S. Morimoto, "Troponin: regulatory function and disorders," Biochemical and Biophysical Research Communications, vol. 369, no. 1, pp. 62-73, 2008.

[33] J. M. Metzger and M. V. Westfall, "Covalent and noncovalent modification of thin filament action: the essential role of troponin in Cardiac muscle regulation," Circulation Research, vol. 94, no. 2, pp. 146-158, 2004.

[34] J. Layland, R. J. Solaro, and A. M. Shah, "Regulation of cardiac contractile function by troponin I phosphorylation," Cardiovascular Research, vol. 66, no. 1, pp. 12-21, 2005.

[35] M. D. Bootman and M. J. Berridge, "The elemental principles of calcium signaling," Cell, vol. 83, no. 5, pp. 675-678, 1995.

[36] C. A. Tate, M. F. Hyek, and G. E. Taffet, "The role of calcium in the energetics of contracting skeletal muscle," Sports Medicine, vol. 12, no. 3, pp. 208-217, 1991. 
[37] X. Liu, N. Takeda, and N. S. Dhalla, "Troponin I phosphorylation in heart homogenate from diabetic rat," Biochimica et Biophysica Acta, vol. 1316, no. 2, pp. 78-84, 1996.

[38] A. E. Messer, A. M. Jacques, and S. B. Marston, "Troponin phosphorylation and regulatory function in human heart muscle: dephosphorylation of Ser23/24 on troponin I could account for the contractile defect in end-stage heart failure," Journal of Molecular and Cellular Cardiology, vol. 42, no. 1, pp. 247-259, 2007.

[39] L. Li, J. Desantiago, G. Chu, E. G. Kranias, and D. M. Bers, "Phosphorylation of phospholamban and troponin I in $\beta$ adrenergic-induced acceleration of cardiac relaxation," American Journal of Physiology, vol. 278, no. 3, pp. H769-H779, 2000.

[40] Y. Pi, K. R. Kemnitz, D. Zhang, E. G. Kranias, and J. W. Walker, "Phosphorylation of troponin I controls cardiac twitch dynamics: evidence from phosphorylation site mutants expressed on a troponin I-null background in mice," Circulation Research, vol. 90, no. 6, pp. 649-656, 2002.

[41] W. Ningyuan, M. Shinya, A. Masazumi et al., "Sheng-Mai-San is protective against post-ischemic myocardial dysfunction in rats through its opening of the mitochondrial KATP channels," Circulation Journal, vol. 66, no. 8, pp. 763-768, 2002.

[42] Z. Liu, Z. Li, and X. Liu, "Effect of ginsenoside Re on cardiomyocyte apoptosis and expression of Bcl-2/Bax gene after ischemia and reperfusion in rats," Journal of Huazhong University of Science and Technology, vol. 22, no. 4, pp. 305-309, 2002.

[43] X. Q. Yi, T. Li, J. R. Wang et al., "Total ginsenosides increase coronary perfusion flow in isolated rat hearts through activation of PI3K/Akt-eNOS signaling," Phytomedicine, vol. 17, no. 13, pp. 1006-1015, 2010.

[44] S. H. Lo, K. S. Lee, L. J. Chen, J. T. Cheng, and C. H. Chen, "Increase of PPAR $\delta$ by dopamine mediated via DA1 receptor-linked phospholipase $\mathrm{C}$ pathway in neonatal rat cardiomyocytes," Autonomic Neuroscience, vol. 177, pp. 211-216, 2013.

[45] Z. C. Chen, K. S. Lee, L. J. Chen, L. Y. Wang, H. S. Niu, and J. T. Cheng, "Cardiac peroxisome proliferator-activated receptor $\delta(\operatorname{PPAR} \delta)$ as a new target for increased contractility without altering heart rate," PLoS One, vol. 8, Article ID e64229, 2013. 

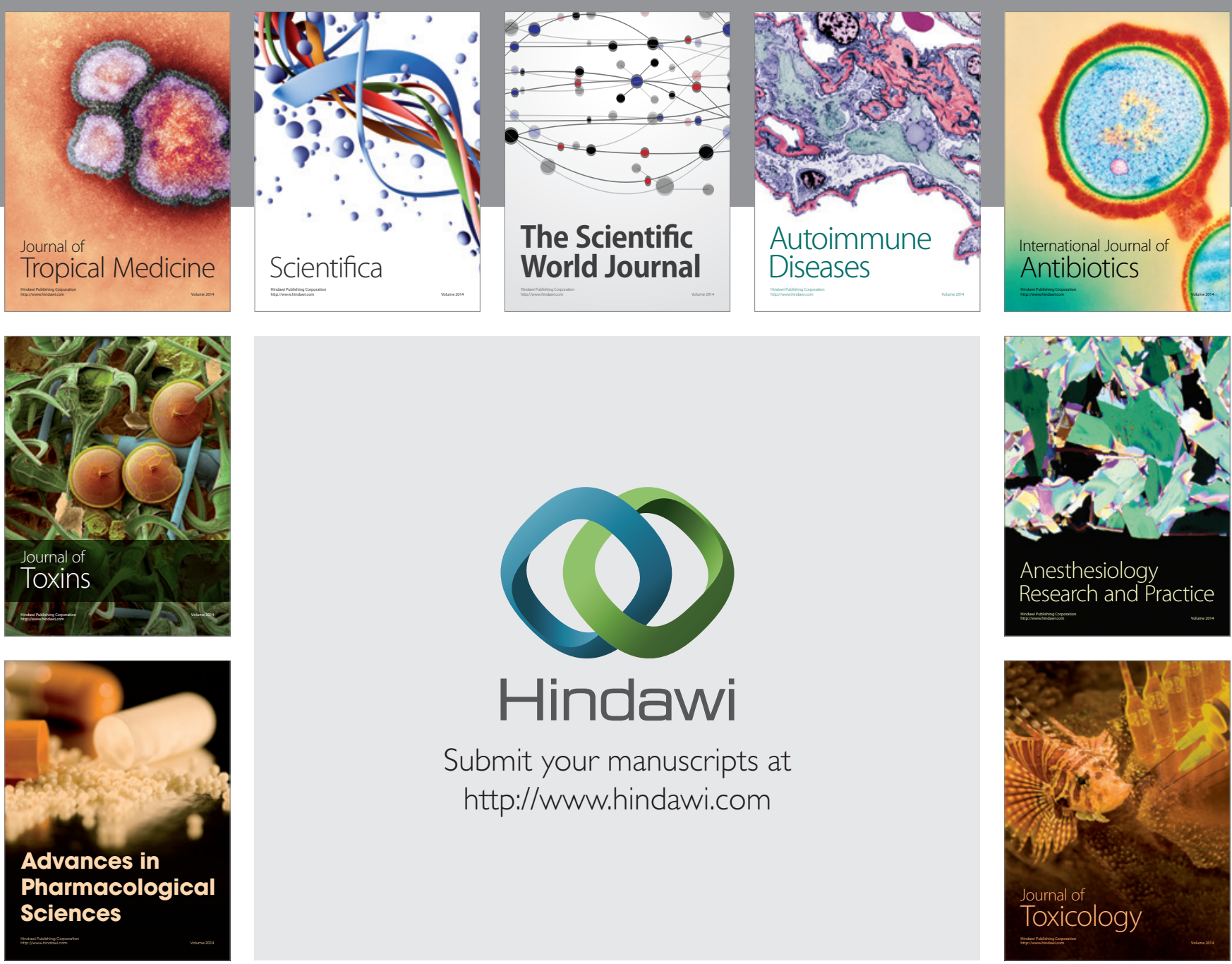

\section{Hindawi}

Submit your manuscripts at

http://www.hindawi.com
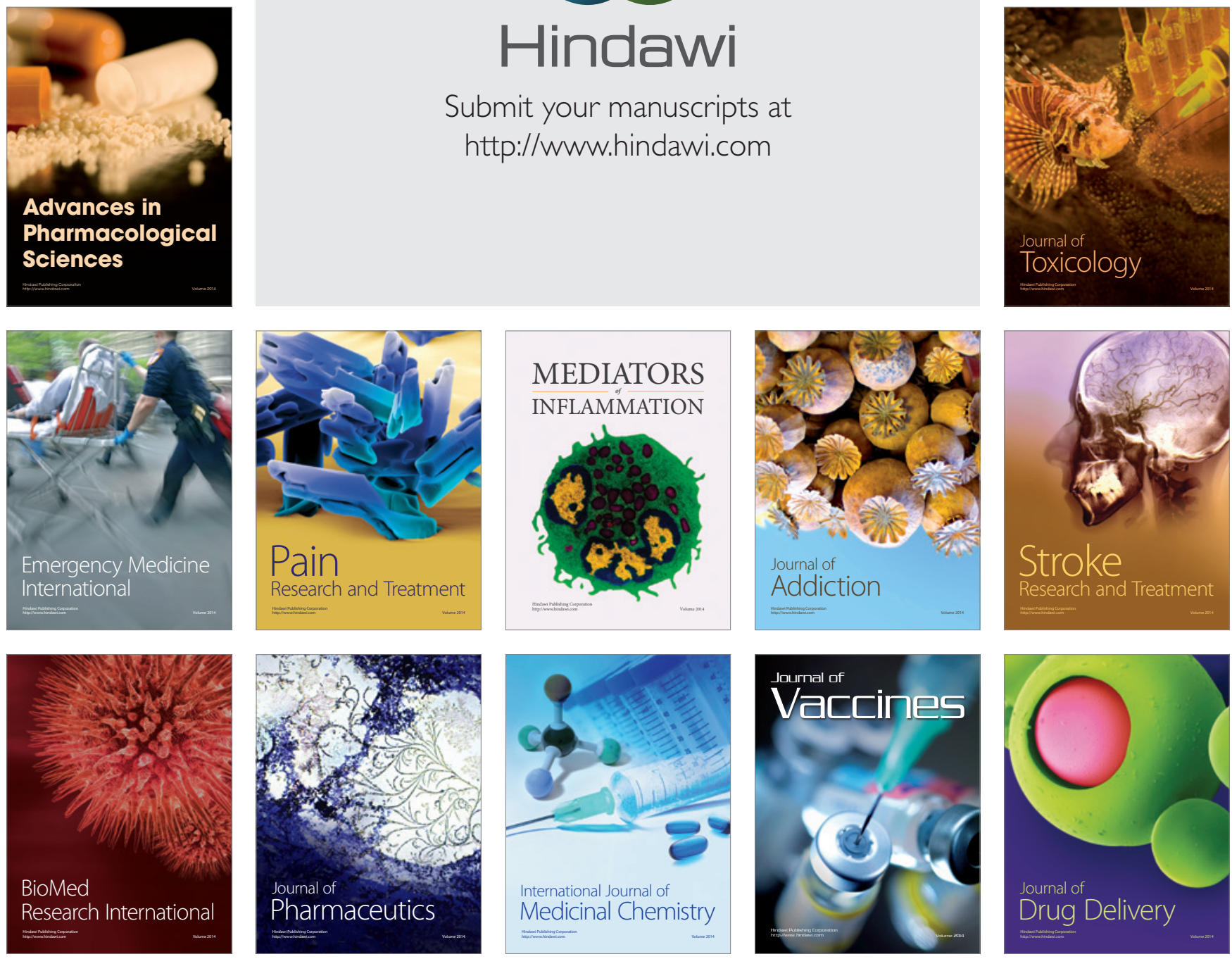